\title{
Aplicação do processo de enfermagem baseado na teoria de Orem ao indivíduo com esclerose múltipla
}

\author{
Application of nursing process based on theory of orem individuals with multiple sclerosis \\ Aplicación del proceso de enfermería basada en la teoría de orem personas con esclerosis múltiple
}

Fernanda Valéria Silva Dantas Avelino, Francinete Paula Silva Dantas Avelino², Railina Laura Uyara

Brandão Sales ${ }^{3}$, Lara Emanueli Neiva de Sousa ${ }^{3}$, Cecília Passos Vaz da Costa ${ }^{3}$

${ }_{1}^{1}$ Doutora em enfermagem, Professora Adjunto da Universidade Federal do Piauí. Teresina, Piauí. Rua Gov. Arthur de Vasconcelos, 2526, Primavera.64.002-530. E:mail.fvdavelino@gmail.com, ${ }^{2}$ Mestre em enfermagem, Professora da Universidade Federal do Piauí. Teresina, Piauí, ${ }^{3}$ Graduanda do Curso de Enfermagem da Universidade Federal do Piauí. Teresina, Piauí

Submissão: 05/01/2011

Aprovação: 14/03/2011

\begin{abstract}
RESUMO
A Esclerose Múltipla (EM) é uma patologia neurológica de caráter autoimune e desmielinizante. Neste trabalho utilizou-se o método do estudo de caso e teve como objetivos: identificar os diagnósticos de enfermagem segundo a Taxonomia da Classificação Internacional das Práticas de Enfermagem (CIPE) e elaborar um plano assistencial tendo a Teoria de Orem como referencial teórico. Os principais diagnósticos de enfermagem encontrados foram: Ansiedade atual, Autocuidado comprometido, Depressão atual, Risco de infecção, Incontinência intestinal atual, Incontinência urinária atual, Mobilidade no leito comprometida, Padrão de higiene comprometido, Padrão de sono comprometido, Pressão sanguínea em nível diminuído, Úlcera por pressão em potencial para aumento. A teoria do autocuidado de Orem é um instrumento válido e possibilitou um planejamento da assistência de enfermagem adequado à problemática do portador de esclerose múltipla.

Descritores: Esclerose múltipla. Teoria de enfermagem. Cuidados de enfermagem.
\end{abstract}

\begin{abstract}
Multiple Sclerosis (MS) is a neurological disease and demyelinating autoimmune character. This work has used the method of case study, and it aimed to identify nursing diagnoses, using therefore the Taxonomy of the International Classification of Nursing Practice (ICNP), and devise a plan of care with the Orem's theory as its theoretical framework. The major nursing diagnoses were: anxiety Current, Self-care compromised, current depression, risk of infection, intestinal current incontinence, urinary incontinence current mobility in bed engaged, committed standard of hygiene, impaired sleep pattern, blood pressure level decreased pressure ulcer potential to increase. The self-care theory of Orem is a valid and possible planning of nursing care most appropriate to the problems of patients with multiple sclerosis.

Descriptors: Multiple sclerosis. Nursing theory. Nursing care.
\end{abstract}

RESUMEN

La esclerosis múltiple (EM) es una enfermedad neurológica desmielinizante y de carácter autoinmune. En este trabajo se ha utilizado el método de estudio de caso, y tuvo como objetivo identificar los diagnósticos de enfermería, utilizando tanto la taxonomía de la Clasificación Internacional de la Práctica de Enfermería (CIPE), y elaborar un plan de atención con la teoría de Orem como el marco teórico. Los diagnósticos principales fueron: la ansiedad actual, cuidado de sí mismo en peligro, la depresión actual, el riesgo de infección, la incontinencia intestinal actual, la movilidad actual de la incontinencia urinaria en la cama comprometidos, comprometidos estándar de higiene, el patrón de alteración del sueño, el nivel de presión arterial disminución de úlcera por presión pueden aumentar. La teoría de auto-cuidado de Orem es una planificación válida y posible de los cuidados de enfermería más adecuada a los problemas de los pacientes con esclerosis múltiple.

Descriptores: Esclerosis múltiple. Teoría de enfermería. Atención de enfermería. 
Avelino FVSD, et al

\section{CONSIDERAÇÕES INICIAIS}

A Esclerose Múltipla (EM) é uma patologia neurológica de caráter autoimune e desmielinizante, uma vez que, o sistema imunológico do próprio organismo se dirige contra a bainha de mielina do sistema nervoso central (SNC), promovendo a desmielinização. As razões deste processo permanecem não elucidadas, entretanto, a hipótese viral desponta como a mais aceita entre pesquisadores. Como resultado da desmielinização, surgem lesões (placas) com acúmulo gradual de tecido cicatricial enrijecido (esclerose), pontos que podem ocorrer em diferentes lugares por todo o SNC, característica esta que justifica a denominação "Esclerose Múltipla" ou "Esclerose em placas"(1).

A EM possui diferentes evoluções, sendo classificada em: surto-remissiva, progressiva primária, progressiva secundária, progressiva-recorrente. A EM surto-remissiva possui evolução mais branda, caracterizada por surtos com dormência e/ou formigamento e/ou visão embaçada, sintomas únicos que duram dias ou semanas e desaparecem em seguida. A EM progressiva primária é marcada pela progressão de comprometimento desde seu aparecimento, sem quaisquer estágios ou remissões óbvias, ou somente com platôs ocasionais e pequenas melhoras temporárias. A progressiva secundária corresponde à EM de manifestação inicial como surto-remissiva e, posteriormente, como progressiva. A progressiva-recorrente é a EM de evolução mais grave, de progressão clara de incapacidade desde a manifestação da doença e de recorrência distinta e aguda que pode ou não ser seguida de recuperação(2).

O diagnóstico não é trivial, podendo levar anos de evolução do caso para ser efetivamente fechado, uma vez que a sintomatologia da EM comumente se assemelha com a de outros tipos de doenças do sistema nervoso. Outro fator que torna, além de difícil, tardio o diagnóstico de EM é a importância não dispensada aos sintomas iniciais, o que torna ainda mais distante a procura por orientação médica e consequente tratamento precoce. Não há, atualmente, exames de sangue, de imagem (Ressonância Magnética de Crânio), testes genéticos, exame de Líquor Cefalorraquidiano (LCR) que possam, por si só, prever ou afirmar diagnóstico de EM. Este é clínico, com base no histórico do paciente, na avaliação dos sintomas percebidos e relatados pelo paciente, e na presença de sinais neurológicos detectados durante o exame neurológico(3).

O enfoque principal do tratamento é o controle dos sinais e sintomas neurológicos, para tanto, medicamentos de alto custo são fornecidos pelo Sistema Único de Saúde (SUS), através do Programa de Medicamentos Excepcionais, cujos fármacos são dispensados mediante encaminhamento médico e efetivação do cadastro do cliente, conforme os critérios encontrados no Protocolo Clínico e Diretrizes Terapêuticas, estabelecidos pela Porta- ria SAS/MS n 97, de 22 de março de $2001^{(4)}$.

A assistência ao indivíduo com EM se configura como um processo dinâmico, no qual a Enfermagem está inserida desempenhando papel fundamental, uma vez que, o enfermeiro é um educador em potencial. $O$ (a) enfermeiro (a) realiza as mais diversas funções que seguem desde o estabelecimento do contato com o cliente e sua família. Disponibiliza informações ao médico sobre as condições do cliente para ajuda e aconseIhamento; instrumentalizando-o para o autocuidado extensivo ao seu cuidador quanto às administrações medicamentosas, em particular, acerca da técnica de utilização das vias intramuscular e hipodérmica para administração de interferon; acompanha-o sistematicamente através de visitas domiciliares e contatos telefônicos regulares. Cabe a Enfermagem planejar ações no sentido de promover a assistência, a recuperação, o autocuidado, o bem-estar, minimizando as limitações impostas pela Esclerose Múltipla e otimizando as potencialidades do cliente, da estrutura familiar e suporte social ${ }^{(5-6)}$.

O processo de enfermagem compreende três construtos teóricos - autocuidado, déficit de autocuidado e sistemas de Enfermagem. O autocuidado descreve e explica a prática de cuidados executados pela pessoa portadora de uma necessidade para manter a saúde e o bem-estar. $O$ déficit de autocuidado constitui a essência da Teoria Geral do Déficit de AutoCuidado por delinear a necessidade da assistência de enfermagem. E, por último, os sistemas de Enfermagem descrevem e explicam como as pessoas são ajudadas por meio dos mecanismos compensatório, parcialmente compensatório e apoio-educação.

Em face do exposto, tem-se como objeto de estudo a Sistematização da Assistência de Enfermagem (SAE), enfatizando as etapas que a compõem assim como sua aplicabilidade dentro do contexto do indivíduo com Esclerose Múltipla. Neste sentido, os objetivos traçados são: identificar os diagnósticos de enfermagem, segundo a Taxonomia da Classificação Internacional das Práticas de Enfermagem (CIPE), elaborar plano assistencial no sentido de melhorar as condições de saúde e bem-estar do portador de EM, tendo a Teoria de Orem como referencial teórico. Com este propósito foi definida a questão norteadora: Quais os possíveis diagnósticos e intervenções de enfermagem na assistência ao portador de EM?

\section{METODOLOGIA}

No presente trabalho utilizou-se o método do estudo de caso, considerado um dos mais relevantes tipos de pesquisa qualitativa, utilizando o processo de enfermagem nas suas fases de histórico, diagnóstico de enfermagem, planejamento, intervenção e avaliação dos resultados. Esta categoria de pesquisa permite-nos 
realizar investigações em profundidade, de um indivíduo, grupo, instituição ou unidade social(7).

O Comitê de Ética em Pesquisa da Universidade Federal do Piauí, reconhecido pela Comissão Nacional de Ética em Pesquisa - CONEP/MS, analisou e aprovou através do protocolo de pesquisa 0079.0.045.00010 em 06 de junho de 2010, de acordo com as Diretrizes estabelecidas na Resolução 196/96 e Complementares do Conselho Nacional de Saúde. A seleção do sujeito da pesquisa foi intencional, atendendo os critérios de inclusão: ter diagnóstico clínico de EM; ser associado à Associação Piauiense de Portadores de esclerose múltipla (APPEM); não apresentar transtornos mentais graves, visto que o desequilíbrio cognitivo inviabilizaria a obtenção de dados verdadeiros e seguros; apresentar grau de dificuldade de mobilidade significativo; consentir de modo livre e esclarecido sua participação por meio da assinatura de termo de consentimento livre e esclarecido (TCLE), o qual assegura anonimato e o direito de não concluir a pesquisa, se o desejar.

Os dados foram coletados de julho a setembro de 2010 por ocasião da realização de visitas domiciliares pelas autoras do trabalho, ao sujeito selecionado, após os esclarecimentos ao mesmo quanto à finalidade da pesquisa e obtenção da anuência. Foram coletados dados a partir da anamnese, da observação e do exame físico, guiados por um roteiro de entrevista denominado de Histórico de Enfermagem, fundamentado e adaptado à Teoria do Autocuidado de Orem. Para a operacionalização do processo de enfermagem com base no autocuidado de Orem, foram realizadas seis visitas domiciliares durante três meses, sendo duas visitas por mês. Esta operacionalização seguiu os seguintes passos do processo: $1^{\circ}$ passo - realização do levantamento de dados através da entrevista; $2^{\circ}$ passo - planejamento das ações de enfermagem através do sistema de apoio-educação; $3^{\circ}$ passo - execução e avaliação das ações de enfermagem no sistema escolhido.

Após a coleta de dados, foi iniciado o trabalho de análise das informações coletadas para a elaboração dos possíveis diagnósticos, problemas de saúde inferidos pelo enfermeiro, pelo fator relacionado ou etiológico e pelas características definidoras, dados objetivos e subjetivos agrupados que evidenciaram os diagnósticos de enfermagem. Na identificação dos diagnósticos de enfermagem, foi utilizado o julgamento clínico e o raciocínio diagnóstico, estabelecido nas seguintes etapas: agrupamentos dos dados e comparação dos padrões a teorias e normas ${ }^{(8)}$.

Para se chegar aos diagnósticos, foram empregadas as características definidoras e os fatores relacionados, determinados pela Classificação Internacional das Práticas de Enfermagem (CIPE), uma vez que representa a base para seleção das intervenções e resultados terapêuticos, além do conhecimento e as experiências das autoras na Sistematização da Assistência de Enfermagem (SAE). De posse dos diagnósticos de enfermagem, foram planejadas e definidas as intervenções a serem implementadas no decorrer da assistência, tomando por base a seguinte definição: roteiro diário ou aprazado que coordena a ação da equipe de enfermagem nos cuidados adequados ao atendimento das pessoas com déficit de auto-cuidado(9).

\section{RESULTADOS E DISCUSSÃO}

\section{Histórico de Enfermagem}

J. M. S. N., sexo feminino, 30 anos, negra, natural de José de Freitas/PI, $2^{\circ}$ grau incompleto, pensionista do INSS, $49 \mathrm{Kg}$ de peso, $1,55 \mathrm{~m}$ de altura, solteira, mãe de um filho de 9 anos. Mora com os pais e o filho em casa de alvenaria e piso bruto, pouco iluminada e pouco arejada, com abastecimento público de água, rede de esgoto e fossa séptica. De uma família de 4 irmãos, filha de mãe idosa e hipertensa e de pai idoso, alcoólatra e tabagista, com relacionamento familiar conturbado por conta dos vícios do pai. Portadora de Esclerose Múltipla há 8 anos e membro da APPEM há 5 anos. Relata episódios prévios, queda de um "pé de jambo" aos 13 anos e "resguardo quebrado", como precursores da EM. Em 2002, morava e trabalhava como babá em Brasília/DF, onde tinha uma rotina exaustiva, o que a levou a considerar as primeiras manifestações da EM - dormência, fraqueza e visão turva - como sintomas isolados, passageiros, decorrentes do cansaço do dia-a-dia. Entretanto, os sintomas rapidamente ganharam maiores proporções, determinando tetraplegia e redução da acuidade visual, exacerbação esta que marcou a busca por ajuda médica e, na sequência, o diagnóstico de EM. Ao regressar ao estado de origem, ainda em 2002, iniciou tratamento específico, submetendo-se a incontáveis pulsoterapias (administrações de altas doses de metilprednisolona venosa) e sessões de fisioterapia, obtendo, assim, importante melhora do padrão de mobilidade de modo que, na ocasião, conseguia deambular com auxílio de andador. Esta evolução foi seguida por significativas crises (exacerbação de sinais e sintomas), marcadas pelo surgimento de dormência nos MMII. Em 2004, foi adicionado à terapêutica o uso de Rebif ${ }^{\circledR} 44$ (betainterferona 1-a), administrado 3x/semana por via subcutânea. Cliente relata ocorrência de sua mais recente crise no final de 2008 e identifica como seus principais cuidadores o filho e a mãe. Atualmente, segue com paraplegia e acuidade visual severamente reduzida, e diagnóstico de EM do tipo progressiva secundária.

\section{Evolução de Enfermagem}

09 de julho de 2010 - Segue no $8^{\circ}$ ano de tratamento domiciliar de EM e de complicações decorrentes 
desta, calma, consciente, orientada, contactuante, fási ca, discurso coerente, abertura ocular espontanea, com pupilas isocóricas e fotoreagentes, respondendo a estímulos verbais, táteis e dolorosos, paraplégica, cadeirante, com acuidade visual acentuadamente reduzida. Encontrada em decúbito ventral sob leito domiciliar, afebril, normosfígmico, padrão respiratório satisfatório, em tratamento de Depressão leve com Cloridrato de paroxítona, de Anemia com Sulfato ferroso, de EM com Rebif® 44 (betainterferona 1-a) 3x semana, por via hipodérmica, com reações adversas de febre, cefaléia e fadiga. Relata rotineira hipotensão mediante troca de decúbito. Ausculta pulmonar sem ruídos adventícios, ausculta cardíaca em dois tempos, ritmica e bulhas normofonéticas. Pele hipocorada, extremidades desidratadas, com presença de 4 úlceras por pressão (UPP) de dimensão de $3 \times 3 \mathrm{~cm}, 5 \times 4 \mathrm{~cm}, 4 \times 3 \mathrm{~cm}$ e $4 \times 4 \mathrm{~cm}$, localizadas, respectivamente, nas regiões ilíaca $\mathrm{D}$, trocantérica $\mathrm{E}$, ilíaca E, sacral, todas em estágio III, apresentando moderada quantidade de secreção purulenta. Realizados curativos oclusivos nas UPP pelo filho ou pela mãe da cliente, uma vez ao dia após o banho, utilizando solução fisiológica $0,9 \%$, gazes e esparadrapo. Cuidadores relatam o uso caseiro de caju, pó de ameixa e mel no tratamento das UPP. Abdômen sem massas palpáveis, ruídos hidroaéreos presentes. Aceita dieta por via oral, em média 4 refeições diárias, restrição a feijão uma vez que os cuidadores associam este alimento a episódio prévio de diarréia. Incontinência urinária com utilização de fraldas apenas em ocasião de compromissos fora de casa, diurese em grande volume, de aspecto concentrado e odor forte. Amenorréica há 2 anos, último Papanicolau há 10 anos. Padrão de higiene comprometido, encontrada sob lençóis encharcados de urina. Padrão de sono e repouso comprometidos em decorrência das reações adversas da medicação betainterferona 1-a. Refere hábitos de acompanhar diariamente programação de rádio, nega vícios. Relata a não adaptação ao colchão de ar e a suspensão do tratamento fisioterápico em virtude do surgimento das úlceras por pressão. Queixa-se de tontura, espasmos, e da "sensação de ossos estarem crescendo", impressão relacionada com a perda de peso e evidenciada pela visualização mais evidente das proeminências ósseas. Confessa sentir-se deprimida rotineiramente e questiona este sentimento. Cliente e familiares demonstram pouco conhecimento sobre a patologia e inquietação proporcional diante o quadro clínico atual, além de demonstrarem interesse em obter informações.

Os resultados encontrados foram agrupados segundo conceitos da teoria do autocuidado de Orem e submetidos a uma análise que levou à identificação de diagnósticos de enfermagem e à formulação de um plano de cuidados conforme os quadros 1 e 2.
Quadro 1 - Principais Diagnósticos de Enfermagem baseados na CIPE (Classificação Internacional da Prática de Enfermagem

\begin{tabular}{|c|c|c|}
\hline FOCO & JULGAMENTO & DIAGNÓSTICO \\
\hline Ansiedade & Atual & Ansiedade Atual \\
\hline Autocuidado & Comprometido & $\begin{array}{c}\text { Autocuidado } \\
\text { Comprometido }\end{array}$ \\
\hline Depressão & Atual & Depressão Atual \\
\hline Dor & Atual & Dor Atual \\
\hline Febre & Atual & Febre Atual \\
\hline Infecção & Atual & Infecção Atual \\
\hline Incontinência Intestinal & Atual & $\begin{array}{c}\text { Incontinência } \\
\text { Intestinal atual } \\
\end{array}$ \\
\hline Incontinência Urinária & Atual & $\begin{array}{c}\text { Incontinência Urinária } \\
\text { Atual }\end{array}$ \\
\hline Luto & Atual & Luto Atual \\
\hline Mobilidade no Leito & Comprometida & $\begin{array}{l}\text { Mobilidade no Leito } \\
\text { Comprometida }\end{array}$ \\
\hline Nível de Consciência & Normal & $\begin{array}{c}\text { Nível de Consciência } \\
\text { Normal }\end{array}$ \\
\hline Padrão Alimentar & Comprometido & $\begin{array}{c}\text { Padrão Alimentar } \\
\text { Comprometido }\end{array}$ \\
\hline Padrão de Higiene & Comprometido & $\begin{array}{l}\text { Padrão Higiene } \\
\text { Comprometido }\end{array}$ \\
\hline Padrão de Repouso & Comprometido & $\begin{array}{l}\text { Padrão de Repouso } \\
\text { Comprometido }\end{array}$ \\
\hline Padrão de Sono & Comprometido & $\begin{array}{l}\text { Padrão de Sono } \\
\text { Comprometido }\end{array}$ \\
\hline Pressão Sanguínea & $\begin{array}{l}\text { Potencial para } \\
\text { Diminuição }\end{array}$ & $\begin{array}{c}\text { Pressão Sanguínea em } \\
\text { Potencial para Diminuição }\end{array}$ \\
\hline Úlcera por Pressão & $\begin{array}{l}\text { Potencial para } \\
\text { Aumento }\end{array}$ & \begin{tabular}{|l|} 
Úlcera por Pressão em \\
Potencial para Aumento
\end{tabular} \\
\hline
\end{tabular}

Quadro 2 - Plano de Cuidados de Enfermagem baseado na Teoria do Autocuidado de Orem

\begin{tabular}{|l|l|}
\hline \multicolumn{1}{|c|}{ Intervenção } & \multicolumn{1}{|c|}{ Resultado Esperado } \\
\hline Disponibilizar informações sobre EM & $\begin{array}{l}\text { Melhoria do nível de conhecimento } \\
\text { acerca da patologia }\end{array}$ \\
\hline $\begin{array}{l}\text { Encorajar o diálogo entre o portador } \\
\text { e familiares }\end{array}$ & $\begin{array}{l}\text { Fortalecimento do vínculo entre o } \\
\text { portador e cuidadores (filho e mãe) }\end{array}$ \\
\hline $\begin{array}{l}\text { Monitorar variações do humor e } \\
\text { orientar familiares }\end{array}$ & Manutenção do humor estável \\
\hline Encaminhar ao psiquiatra & Prevenção dos episódios depressivos \\
\hline $\begin{array}{l}\text { Orientar paciente e família sobre } \\
\text { cuidados gerais }\end{array}$ & $\begin{array}{l}\text { Melhoria do manejo na realização } \\
\text { de atividades diárias }\end{array}$ \\
\hline $\begin{array}{l}\text { Orientar colchão de ar como } \\
\text { melhor indicação }\end{array}$ & Prevenção e tratamento de UPP \\
\hline $\begin{array}{l}\text { Supervisionar cicatrização das UPPs } \\
\text { já presentes }\end{array}$ & Finalização do processo de cicatrização \\
\hline $\begin{array}{l}\text { Observar sinais de isquemia em } \\
\text { proeminências ósseas }\end{array}$ & Prevenção do surgimento de UPP \\
\hline $\begin{array}{l}\text { Avaliar nível de consciência, respostas } \\
\text { sensitiva e motora }\end{array}$ & $\begin{array}{l}\text { Avaliação da atrofia muscular e } \\
\text { espasmos }\end{array}$ \\
\hline $\begin{array}{l}\text { Orientar cuidadores quanto ao transporte, } \\
\text { armazenamento e administração do betainterferona-1a }\end{array}$ & $\begin{array}{l}\text { Corretos transporte, armazenamento } \\
\text { e administração medicamentosa }\end{array}$ \\
\hline $\begin{array}{l}\text { Encaminhar ao nutricionista } \\
\text { Melhoria no quadro de anemia e na } \\
\text { cicatrização das UPPs }\end{array}$ \\
\hline $\begin{array}{l}\text { Encaminhar à Equipe de Estratégia } \\
\text { Saúde da Família da região }\end{array}$ & $\begin{array}{l}\text { Promoção e prevenção da saúde da } \\
\text { mulher }\end{array}$ \\
\hline $\begin{array}{l}\text { Solicitar visita e acompanhamento } \\
\text { da equipe multidisciplinar da APPEM }\end{array}$ & Melhoria da adesão ao tratamento \\
\hline
\end{tabular}


Um estudo realizado mediante elaboração de um instrumento de coleta de dados, fundamentado no modelo conceitual de Orem, evidenciou a importância do processo de sistematização da coleta de dados no sentido de elaborar plano de cuidados e oferecer uma assistência de qualidade ${ }^{(9-10)}$. Esses achados corroboram com nosso estudo no qual a SAE foi embasada na teoria de Orem, proporcionando um alicerce no levantamento dos dados do cliente de forma a oferecer o cuidado integral e de qualidade.

O autocuidado (AC) inclui a ação, a capacidade e as exigências terapêuticas de autocuidado, bem como os seus requisitos. O AC é a prática de atividades executadas pelos indivíduos, em seu próprio benefício, para a manutenção da vida, da saúde e do bem-estar, que depende de fatores internos e externos ao indivíduo, como: idade, sexo, estado de saúde, fatores socioculturais, padrão de vida, disponibilidade de recursos, entre outros. O déficit de AC é a condição e circunstância que colocam as pessoas em situações de carência de cuidados de saúde. Os conceitos fundamentais dessa teoria são: agente de autocuidado, demanda de autocuidado e déficit de autocuidado ${ }^{(8)}$. A teoria de Orem explicita bem esses conceitos, além de reforçar que a enfermagem deve utilizar estratégias que venham reduzir esse déficit de autocuidado, mas para isso é necessário que a enfermeira tenha conhecimento técnico-cientifico associado ao conhecimento da realidade na qual o portador de EM está inserido.

\section{CONSIDERAÇÕES FINAIS}

Em face do exposto é possível considerar que o uso da teoria do autocuidado de Dorothea Orem é um instrumento válido, o qual nos ajudou a promover uma comunicação mais objetiva entre pesquisador e

\section{REFERÊNCIAS}

1. Gerald Filho B. Patologia. 7 ed. Rio de Janeiro:Guanabara Koogan, 2006.

2.Kalb RC. Esclerose Múltipla: perguntas e respostas. São Paulo (SP): Atlas; 2000.

3.Grzesiuk AK. Características Clínicas e Epidemiológicas de 20 Pacientes Portadores de Esclerose Múltipla Acompanhados em Cuiabá-Mato Grosso. Arq Neuro-Psiquiatria 2006;64(3):635-38

4. Ministério da Saúde (BR). Protocolo Clínico e Diretrizes Terapêuticas dos Medicamentos Excepcionais. Secretaria de Assistência à Saúde. Brasília: Ministério da Saúde; 2002.

5.Almeida LHRB de, Rocha FC, Nascimento FCL, pesquisada, adequando-se de certa forma ao planejamento da assistência de enfermagem, à problemática do portador de Esclerose Múltipla. Considera-se também, que o processo de enfermagem baseado em Orem, nos deu subsídios para a aplicação sistemática dessa assistência, fazendo-se mudanças necessárias ao plano de cuidados mesmo.

Dessa forma, diante dos resultados obtidos no estudo de caso, os diagnósticos de enfermagem encontrados foram: Ansiedade atual, Autocuidado comprometido, Depressão atual, Dor atual, Febre atual, Infecção Atual, Incontinência intestinal atual, Incontinência urinária atual, Luto atual, Mobilidade no leito comprometida, Nível de consciência normal, Padrão alimentar comprometido, Padrão de higiene comprometido, Padrão de repouso comprometido, Padrão de sono comprometido, Pressão sanguínea em nível diminuído, Úlcera por pressão em potencial para aumento. Estes diagnósticos evidenciam as incapacidades para a resolução de seus problemas atuais, de alcance e desempenho de papéis desejáveis e de mudanças na sua vida social, as quais reforçam a importância da utilização dos construtos teóricos da Teoria de Orem.

Embora este trabalho represente um estudo preliminar no levantamento de possíveis diagnósticos de enfermagem identificados em um indivíduo com EM, o mesmo nos permitiu entender que o diagnóstico de enfermagem não pode ser uma fase isolada de todo o processo assistencial, mas, que deve ser utilizado com o objetivo de direcionar a ação de enfermagem e à medida em que as ações do planejamento do cuidado forem executadas, faz-se necessário um contínuo processo de avaliação. Cabe ressaltar, ainda, a necessidade de outros estudos nessa área junto a clientes com EM, para a definição de um perfil diagnóstico baseado na CIPE, a fim de subsidiar a assistência de enfermagem sistematizada e fundamentada cientificamente .

Campelo LM. Ensinando e aprendendo com portadores de Esclerose Múltipla: relato de experiência. Rev bras enferm 2007; 60(4):460-3

6. George JB. Teorias de Enfermagem. Porto Alegre: Artes Médicas, 1993.

7. Yin RK. Estudo de caso: planejamento e métodos. 2 ed. Porto Alegre: Bookman; 2001.

8. Johnson MMAAS. Classificação de Resultados de Enfermagem. 2 ed. Porto Alegre: Artmed; 2004.

9. Orem DE. Nursing: concepts of practice. 6 th ed. St Louis (USA): Mosby Inc.; 2001.

10. Luney M. Pensamento crítico e diagnóstico de enfermagem estudo de caso e análise. Porto Alegre: Artmed; 2004. 